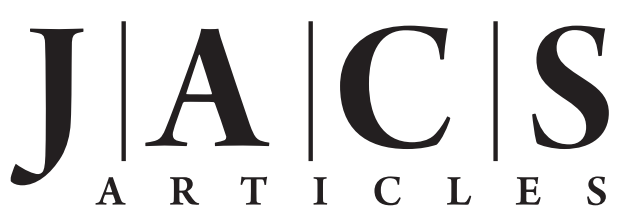

Published on Web 06/04/2010

\title{
NMR Methods for Characterizing the Pore Structures and Hydrogen Storage Properties of Microporous Carbons
}

\author{
Robert J. Anderson, ${ }^{\dagger}$ Thomas P. McNicholas, ${ }^{\ddagger}$ Alfred Kleinhammes, ${ }^{*, \dagger}$ \\ Anmiao Wang, ${ }^{\ddagger}$ Jie Liu, ${ }^{\ddagger}$ and Yue $\mathrm{Wu}^{\dagger}$ \\ Department of Physics and Astronomy, University of North Carolina, Chapel Hill, \\ North Carolina 27514-3255, and Department of Chemistry, Duke University, \\ Durham, North Carolina 27708
}

Received January 6, 2010; E-mail: kleinham @ physics.unc.edu

\begin{abstract}
H}$ NMR spectroscopy is used to investigate a series of microporous activated carbons derived from a poly(ether ether ketone) (PEEK) precursor with varying amounts of burnoff (BO). In particular, properties relevant to hydrogen storage are evaluated such as pore structure, average pore size, uptake, and binding energy. High-pressure NMR with in situ $\mathrm{H}_{2}$ loading is employed with $\mathrm{H}_{2}$ pressure ranging from $100 \mathrm{~Pa}$ to $10 \mathrm{MPa}$. An $\mathrm{N}_{2}$-cooled cryostat allows for NMR isotherm measurements at both room temperature $(\sim 290 \mathrm{~K})$ and $100 \mathrm{~K}$. Two distinct ${ }^{1} \mathrm{H}$ NMR peaks appear in the spectra which represent the gaseous $\mathrm{H}_{2}$ in intergranular pores and the $\mathrm{H}_{2}$ residing in micropores. The chemical shift of the micropore peak is observed to evolve with changing pressure, the magnitude of this effect being correlated to the amount of $\mathrm{BO}$ and therefore the structure. This is attributed to the different pressure dependence of the amount of adsorbed and non-adsorbed molecules within micropores, which experience significantly different chemical shifts due to the strong distance dependence of the ring current effect. In pores with a critical diameter of $1.2 \mathrm{~nm}$ or less, no pressure dependence is observed because they are not wide enough to host non-adsorbed molecules; this is the case for samples with less than 35\% BO. The largest estimated pore size that can contribute to the micropore peak is estimated to be around $2.4 \mathrm{~nm}$. The total $\mathrm{H}_{2}$ uptake associated with pores of this size or smaller is evaluated via a calibration of the isotherms, with the highest amount being observed at 59\% BO. Two binding energies are present in the micropores, with the lower, more dominant one being on the order of $5 \mathrm{~kJ} \mathrm{~mol}^{-1}$ and the higher one ranging from 7 to $9 \mathrm{~kJ} \mathrm{~mol}^{-1}$.
\end{abstract}

\section{Introduction}

Nuclear magnetic resonance (NMR) has now been used extensively in adsorption and surface studies for several decades. ${ }^{1}$ However, NMR of molecular hydrogen has rarely been utilized as a tool for probing nanostructures. In fact, $\mathrm{H}_{2}$ is even rarely used for surface area determination due to an inconveniently low condensation point of $20.28 \mathrm{~K}$ which is experimentally required for the application of the commonly used Brunauer-Emmett-Teller (BET) theory. ${ }^{2}$ With the increasing interest in vehicular hydrogen storage, it is crucial that the potential applications of a technique as versatile and powerful as NMR are not overlooked for hydrogen storage studies. One method proposed to meet the U.S. Department of Energy (DOE)'s goals ${ }^{3}$ for safe and efficient hydrogen storage

University of North Carolina.

Duke University.

(1) Turov, V. V.; Leboda, R. In ${ }^{1} H$ NMR Spectroscopy of Adsorbed Molecules and Free Surface Energy of Carbon Adsorbents; Radovic, L. R., Ed.; Chemistry and Physics of Carbon 27; Marcel-Dekker: New York, 2001; pp 67-124.

(2) Brunauer, S.; Emmett, P. H.; Teller, E. J. Am. Chem. Soc. 1938, 60 309-319.

(3) DOE Office of Energy Efficiency and Renewable Energy. Hydrogen, Fuel Cells \& Infrastructure Technologies Program Multi-Year Research, Development and Demonstration Plan. http://www.eere.energy. gov/hydrogenandfuelcells/mypp/pdfs/storage.pdf (accessed October 14, 2009). is physisorption in porous carbon-based materials. ${ }^{4}$ Since hydrogen is the adsorbate species in this case, it would be advantageous and quite appropriate to use it to probe the nanostructures of these materials, whereas the traditional probe molecules $\left(\mathrm{N}_{2}, \mathrm{CO}_{2}\right.$, etc.) may not be able to fully explore all of the $\mathrm{H}_{2}$-relevant pore structures and surfaces due to issues such as differences in accessibility and molecular dynamics. Further, the lower limit of pore size where the BET approach is applicable for determining pore size distributions is uncertain, and some have suggested this to be as large as $7.5 \mathrm{~nm} .{ }^{5}$ For hydrogen storage, in addition to requiring a large specific surface area (SSA) for maximum hydrogen uptake, the sorbent material also needs to be almost exclusively microporous $(d<2 \mathrm{~nm}){ }^{6}$ and thus a method which is better equipped for probing the smallest of pores is needed. Recently, ${ }^{1} \mathrm{H}$ and ${ }^{2} \mathrm{H}$ NMR was used to evaluate the hydrogen storage properties of carbon

(4) Dillon, A. C.; Heben, M. J. Appl. Phys. A: Mater. Sci. Process. 2001, $72,133-142$.

(5) Lastoskie, C. M.; Gubbins, K. E. Characterization of Porous Materials Using Density Functional Theory and Molecular Simulation In Characterisation of porous solids V; Proceedings of the 5th International Symposium on the Characterisation of Porous Solids (COPSV), Heidelberg, Germany,, May 30-June 2, 1999; Unger, K. K., Kreysa, G., Baselt, J. P., Eds.; Studies in Surface Science and Catalysis 128; Elsevier: Amsterdam, 2000; pp 41-50.

(6) Bhatia, S. K.; Myers, A. L. Langmuir 2006, 22, 1688-1700. 
Table 1. Samples Characterized by NMR in This Paper

\begin{tabular}{lccc}
\hline \multicolumn{1}{c}{ sample } & mass $(\mathrm{mg})$ & density $\left(\mathrm{g} \mathrm{cm}^{-3}\right)$ & BET surface area $\left(\mathrm{m}^{2} \mathrm{~g}^{-1}\right)$ \\
\hline CO2-9-1 & 138.3 & 0.795 & 524 \\
CO2-9-26 & 81.3 & 0.467 & 1027 \\
CO2-9-59 & 65.1 & 0.374 & 1986 \\
CO2-9-80 & 41.3 & 0.237 & 3103 \\
Steam-9-20 & 109.6 & 0.630 & 1294 \\
Steam-9-35 & 86.8 & 0.499 & 981 \\
Steam-9-47 & 56.9 & 0.327 & 1207 \\
Steam-9-70 & 50.6 & 0.291 & 1956 \\
Steam-9-95 & 19.8 & 0.114 & 2802
\end{tabular}

nanotubes $;^{7}$ adsorption isotherms based on the NMR intensity were obtained, but no method for calibrating the absolute quantities was proposed. Such isotherms, properly converted to units of mass, would be a useful alternative to volumetric and gravimetric isotherms. Furthermore, in carbonaceous samples chemical shifts arising from the ring-current effect $^{8}$ could potentially allow the $\mathrm{H}_{2}$ occupying intergranular macropores to be distinguished from $\mathrm{H}_{2}$ residing in smaller pores, providing valuable information about nanostructures which the other methods for producing isotherms are not able to give. In addition to the uptake of the smaller pores, the binding energies associated with their surfaces may also be determined from this information, both of which are important parameters for the application of hydrogen storage, with a DOE 2015 target value ${ }^{3}$ of $9 \mathrm{wt} \%$ for the uptake and a calculated ideal range of 20-40 $\mathrm{kJ} \mathrm{mol}^{-1}$ for the binding energy. ${ }^{9}$ Since it is crucial that candidate materials can be engineered to have optimal pore sizes and structures if physisorption is to be a viable option for vehicular storage,$^{10}$ the information about the structure provided by the NMR method will prove to be valuable feedback for materials synthesis. In this work, methods for applying basic NMR techniques to hydrogen storage studies are presented, with a family of activated carbons derived from poly(ether ether ketone) (PEEK) serving to demonstrate the capabilities made available by such methods.

\section{Experimental Details}

2.1. Sample Preparation. Pellets of PEEK were preactivated under an argon flow of $700 \mathrm{sccm}$ at $900{ }^{\circ} \mathrm{C}$ for $30 \mathrm{~min}$, followed by activation by a $100 \mathrm{sccm}$ flow of either steam or $\mathrm{CO}_{2}$ at $900{ }^{\circ} \mathrm{C}$ for varying amounts of time. The purpose of the preactivation was to carbonize the material, while the activation served to develop the microporous structure by attacking the weak bonds in the carbonized PEEK created by the first step. Complete details of the synthesis procedure can be found elsewhere ${ }^{11}$ along with more comprehensive sample analysis. The sample nomenclature is activation method-9-percent burn off, where 9 indicates the activation temperature of $900{ }^{\circ} \mathrm{C}$ and the burnoff (BO) is relative to the mass after the preactivation step. For example, PEEK steamactivated at $900{ }^{\circ} \mathrm{C}$ with $70 \% \mathrm{BO}$ is labeled Steam-9-70. Table 1 lists the nine samples studied in this work along with their mass filling the NMR coil detection region $\left(V=0.174 \mathrm{~cm}^{3}\right)$, the corresponding packing density, and BET surface area, the latter having been measured at the National Renewable Energy Laboratory. ${ }^{11}$

2.2. Sample Characterization. ${ }^{1} \mathrm{H}$ NMR spectra were recorded using a basic free induction decay pulse sequence in a $4.7 \mathrm{~T}$

(7) Shen, K.; Pietrass, T. J. Phys. Chem. B 2004, 108, 9937-9942.

(8) Gradsztajn, S.; Conard, J.; Benoit, H. J. Phys. Chem. Solids 1970, 31, $1121-1135$

(9) Lochan, R. R. C.; Head-Gordon, M. Phys. Chem. Chem. Phys. 2006, $8,1357-1370$.

(10) Nijkamp, M. G.; Raaymakers, J. E. M. J.; van Dillen, A. J.; de Jong, K. P. Appl. Phys. A: Mater. Sci. Process. 2001, 72, 619.

(11) McNicholas, T. P.; Anderson, R. J.; Kleinhammes, A.; Wang, A.; Wang, Y.; Wu, Y.; Liu, J. J. Phys. Chem. A 2010, submitted. superconducting magnet. The PEEK samples were subject to $\mathrm{H}_{2}$ gas in situ at $\mathrm{H}_{2}$ pressures ranging from $100 \mathrm{~Pa}$ to $10 \mathrm{MPa}$, at both room temperature $(\mathrm{RT}, \sim 290 \mathrm{~K})$ and $100 \mathrm{~K}$. The shift of the free $\mathrm{H}_{2}$ gas peak observed for each specific sample is used as the shift reference in ppm, the reasons for which will be discussed later. A custom-built probe which allowed for in situ pressure adjustment within a cylindrical sapphire sample holder (inner diameter of 1.7 $\mathrm{mm}$ and outer diameter of $3 \mathrm{~mm}$ ) was placed inside a nitrogen flow cryostat to achieve the desired pressure and temperature conditions. Three pressure gauges were used in order to accurately cover a range from $0.13 \mathrm{~Pa}$ to $12 \mathrm{MPa}$. A pressure gauge accurate below $50 \mathrm{kPa}$ was added to the experimental setup after several samples had already been characterized. Isotherms for CO2-9-59 and Steam-9-20, -47, -70, and -95 were measured over the full pressure range, while CO2-9-1, -26, and -80 and Steam-9-35 were probed between 0.1 and $10 \mathrm{MPa}$. In all cases, the sample was dynamically evacuated at RT for $12 \mathrm{~h}$ to less than $1 \mathrm{~Pa}$ using a turbopump before the initial loading of hydrogen. NMR spectra were taken during the initial pump-down and the removal of $\mathrm{H}$-containing contaminants, mainly $\mathrm{H}_{2} \mathrm{O}$, adsorbed on the sample was observed. After pumping down, a background spectrum was obtained. The activated PEEK samples appear remarkably clean based on their proton background. Samples with BO larger than $35 \%$ do not show a proton background measurable by NMR. The background was subtracted from the presented spectra. All background spectra taken after completed isotherm measurements were identical to those taken in the pumped state at the beginning of the experiments, indicating that no permanent $\mathrm{H}$ bonds were formed during the hydrogen exposure of the samples. After the background spectrum was recorded, a spectral isotherm up to $10 \mathrm{MPa}$ was measured for calibration purposes. The system was then cooled to $100 \mathrm{~K}$ while still under pressure. Once $100 \mathrm{~K}$ had been maintained for a minimum of $15 \mathrm{~min}$, the pressure was readjusted to $10 \mathrm{MPa}$ and another isotherm was acquired, this time decreasing in pressure. Afterward, a background spectrum was taken once the system had been dynamically pumped for an average of $15 \mathrm{~min}$. Focusing primarily on the desorption isotherm at $100 \mathrm{~K}$ can be justified since hysteresis effects are expected to be minimal so far above the critical temperature of molecular hydrogen. All samples exhibited fast kinetics even at $100 \mathrm{~K}$, and an equilibrium pressure was arrived at in $20-30 \mathrm{~s}$ or less after changing the system pressure. The average time available for the system to approach equilibrium between measurements ranged between 52 and $165 \mathrm{~s}$, making nonequilibrium scenarios unlikely.

\section{Results and Discussion}

3.1. Relationship of NMR Spectra and Pore Structure. The ${ }^{1} \mathrm{H}$ NMR spectra at $100 \mathrm{~K}$ of CO2-9-59 as they evolve with pressure are shown in Figure 1 (not all pressures are represented here for the sake of clarity). Two distinct peaks, A on the left and $\mathrm{B}$ on the right, are clearly resolved and evolve very differently as the pressure is varied. Peak A increases linearly with pressure, consistent with the behavior of a free gas, and is not clearly visible until around $0.3 \mathrm{MPa}$. In contrast, peak B is present even at $100 \mathrm{~Pa}$ but eventually approaches saturation at higher pressure, consistent with Type I monolayer surface adsorption. Interestingly, in Figure 2 the chemical shifts of peak $\mathrm{B}$ can be observed moving closer to peak $\mathrm{A}$ as the pressure is increased, a behavior which is even more obvious when the position of peak B is plotted versus a log scale of the pressure (inset). An intriguing trend appears when the varying chemical shift is plotted for all nine samples (Figure 3): the strength of this pressure dependence increases with the amount of $\mathrm{BO}$ but is absent below 35\% BO! This suggests that the NMR method is sensitive to changes in the structure of the materials and can 


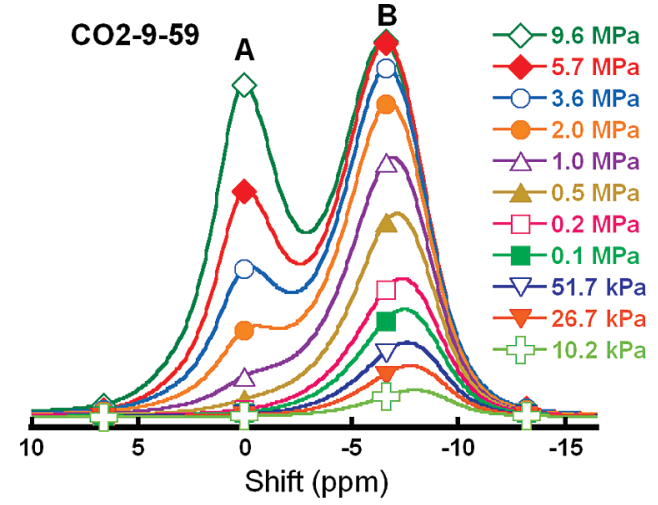

Figure 1. Evolution of the NMR spectra as a function of pressure for $\mathrm{CO} 2-$ 9-59 at $100 \mathrm{~K}$. Peak A on the left evolves linearly with pressure and does not have a significant contribution at low pressures, while peak B is present even at the lowest pressure and approaches saturation at higher pressures.

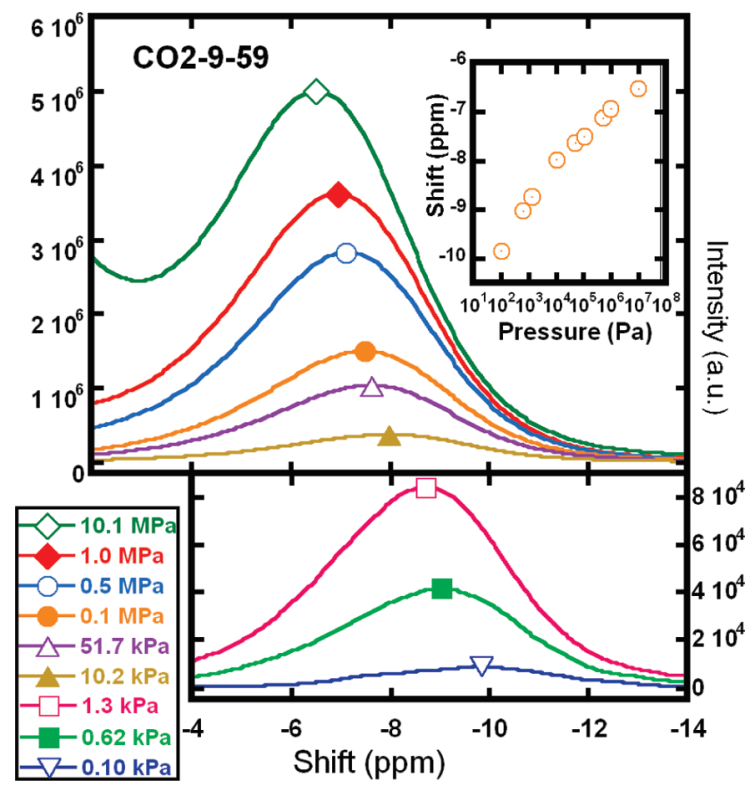

Figure 2. Spectra of $\mathrm{H}_{2}$ in $\mathrm{CO} 2-9-59$ at $100 \mathrm{~K}$, demonstrating a pressure dependence in the chemical shift of peak B. The maximum of peak B is indicated by each spectrum's respective marker, ranging from about -10 $\mathrm{ppm}$ at the lowest pressures to approximately $-6 \mathrm{ppm}$ at $10 \mathrm{MPa}$. The chemical shift is plotted against pressure with a logarithmic axis (inset) to emphasize this dynamic behavior.

provide information useful for engineering them for optimal hydrogen storage.

3.2. Chemical Shift and Pore Dimensions. A ${ }^{1} \mathrm{H}$ nucleus in a given chemical environment will, when exposed to a uniform magnetic field, experience an induced magnetic field, giving rise to unique NMR resonance reflecting that chemical bond-the chemical shift. Experimentally, the resonances of various nuclei with different resonance frequencies are measured with respect to a reference compound. The chemical shift, defined as $\delta=$ $\left(v_{\text {nucl }}-v_{\text {ref }}\right) / \nu_{\text {ref }}$, is measured in relative units of ppm $\left(10^{-6}\right)$. For ${ }^{1} \mathrm{H}$ the standard reference is tetramethylsilane $\left[\mathrm{Si}\left(\mathrm{CH}_{3}\right)_{4}\right.$, or TMS], which is given a value of zero. The chemical shift convention assigns ${ }^{1} \mathrm{H}$ nuclei resonating downfield (to the left) of TMS positive values and regards those as less shielded, while nuclei resonating to the right of TMS are considered more shielded or diamagnetic and given negative chemical shift values. Hydrogen gas was measured at $6.9 \mathrm{ppm}$, liquid $\mathrm{H}_{2} \mathrm{O}$ at $4.8 \mathrm{ppm}$, and gaseous $\mathrm{H}_{2} \mathrm{O}$ at $0.4 \mathrm{ppm}$. NMR lines of ${ }^{1} \mathrm{H}$ nuclei associated with $\mathrm{C}-\mathrm{H}$ bonds, in chains, aromatic compounds,

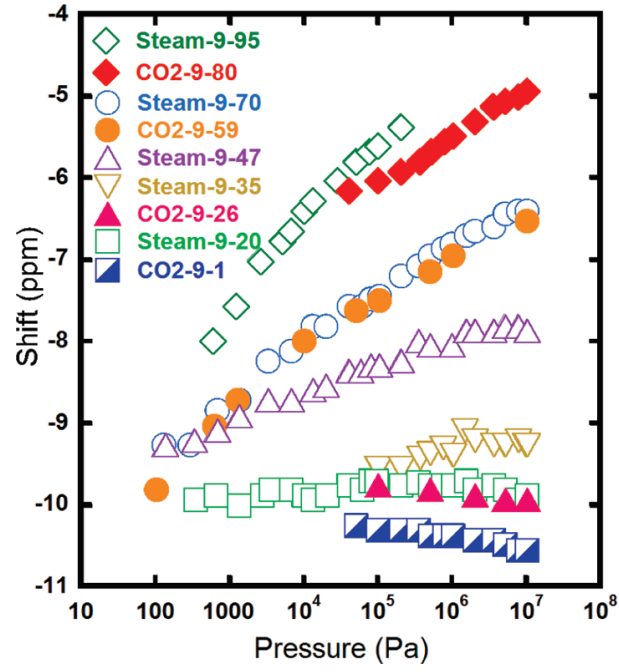

Figure 3. Chemical shift of the micropore peak versus pressure at $100 \mathrm{~K}$. A noticeable change in shift with varied pressure is expected only in samples whose average pore size is larger than $\sim 1.2 \mathrm{~nm}$. All nine samples appear to have the same low-pressure limit of about $-10 \mathrm{ppm}$, indicating little variation in the degree of graphitization of the adsorbent surface. The error of the shift is approximately $\pm 0.2 \mathrm{ppm}$ and is represented by the size of the data points.

etc., are found to the left of TMS, with ${ }^{1} \mathrm{H}$ associated with alkane chains being located between 1 and $2 \mathrm{ppm}$ and the proton line of benzene at 7.26 ppm. ${ }^{12}$ The difference in chemical shift between the ethylenic and aromatic protons was first explained by Pople, ${ }^{13}$ who invoked an induced ring current extending along the benzene ring and involving the $\pi$ electrons. Here, the protons are located in the plane of the benzene ring, and the ring current effect affords an additional downfield shift that adds to the ethylenic (or local) contribution. ${ }^{13}$ A hypothetical ${ }^{1} \mathrm{H}$ nucleus located above the center of the benzene ring would show a diamagnetic response due to the ring current and the resonance would be shifted upfield, the ${ }^{1} \mathrm{H}$ nucleus being more shielded (see Figure 4). In fact, the measured and simulated upfield chemical shift of protons within aromatic compounds is used to gauge the aromaticity of the compound. Consequently, the chemical shift for a hydrogen molecule adsorbed on graphene and located above the plane of the aromatic rings is expected to be strongly diamagnetic. Indeed, the chemical shift for line B is upfield of TMS and clearly not related to conventional $\mathrm{C}-\mathrm{H}$ bonds.

Today the Pople-Langston-Pauling approach of invoking ring currents has been replaced by ab initio quantum mechanical computations. However, the intuitive picture of the extended ring current and induced dipole moment giving rise to a local field at the probe nucleus is still useful ${ }^{14}$ and is invoked here in a phenomenological approach. The chemical shift tensor due to the locally induced ring currents alone can be estimated as ${ }^{15}$

$$
\sigma_{x x}=\sigma_{y y} \cong-\frac{1}{4 \pi} \chi_{\perp} \frac{1}{z^{3}} ; \quad \sigma_{z z} \cong 2 \frac{1}{4 \pi} \chi_{\|} \frac{1}{z^{3}}
$$

Here, $z$ is the vertical distance to the center of the probe molecule from the center of the plane, and $\chi_{\perp}$ and $\chi_{\|}$are the molar

(12) Schmidt-Rohr, K.; Spiess, H. W. Multidimensional Solid-State NMR and Polymers; Academic Press, Inc.: San Diego, 1994; p 39.

(13) Pople, J. A. J. Chem. Phys. 1956, 24, 1111.

(14) Haddon, R. C. Nature 1995, 378, 249-255.

(15) Carrington, A. In Introduction to Magnetic Resonance with Applications to Chemistry and Chemical Physics; McLachlan, A. D., Ed.; Harper \& Row: New York, 1967; pp 58-60. 


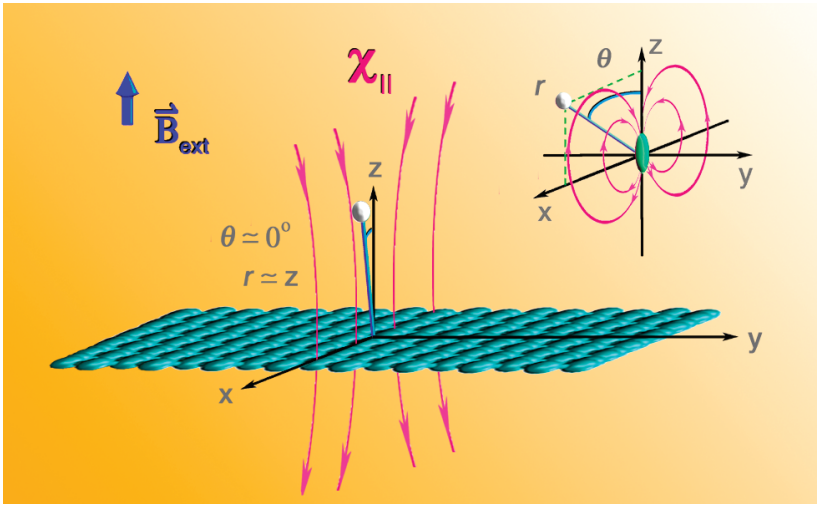

Figure 4. Upper right: Normally, a molecular grouping with an axially symmetric susceptibility tensor has tensor components which are dependent on $r$, the radial distance of the probe molecule from the center of the grouping; $\theta$, which is the angle between the $z$-axis and the location of the probe molecule in the $x-z$ plane; and $\chi_{\perp}$ and $\chi_{\|}$, which are the molar magnetic susceptibilities of the grouping perpendicular and parallel to the $z$-axis, respectively ( $\chi_{\|}$shown here). Center: For a $\mathrm{H}_{2}$ molecule adsorbed above a graphene plane and experiencing a local field due to the locally induced dipoles in the plane, $\theta \approx 0^{\circ}$ and $r \approx z$, corresponding to the vertical distance from the plane.

magnetic susceptibilities of the material perpendicular and parallel to the $z$-axis, respectively. Complete details of this approximation can be found in Appendix A in the Supporting Information. A tensor similar to eq 1 gives rise to a powder pattern due to an isotropic distribution of orientations of the plane with respect to the external magnetic field. ${ }^{16}$ However, as discussed in Appendix B in the Supporting Information, the molecular kinetics are fast with respect to the NMR time scale ( $\sim 3 \mathrm{~ms}$ ), and thus only a single, [approximately] symmetric peak will be observed, centered at the average of the three tensor components:

$$
\delta=\frac{2}{3} \frac{1}{4 \pi}\left(\chi_{\|}-\chi_{\perp}\right) \frac{1}{z^{3}}
$$

Here, the quantity $\left(\chi_{\|}-\chi_{\perp}\right)$ is also known as the total anisotropy of the susceptibility $\chi_{\text {Tot }}$ and has been experimentally determined $^{17}$ to be approximately $-4.4 \times 10^{-9} \mathrm{~m}^{3} \mathrm{~mol}^{-1}$ at $T=$ $100 \mathrm{~K}$ for graphite. Using the same phenomenological approach, Tabony ${ }^{18}$ estimated the chemical shift observed for molecules adsorbed on graphite at $z=0.3 \mathrm{~nm}$ to be $-35 \mathrm{ppm}$, consistent with his experimental value of $-37 \mathrm{ppm}$ determined for protons of aromatic compounds adsorbed above a graphitic plane at low coverage and measured with respect to the value found in the respective liquid. However, for $\mathrm{H}_{2} \mathrm{O}$ adsorbed on graphite, Tabony ${ }^{18}$ measured an upfield shift of approximately $-15 \mathrm{ppm}$ with respect to water in intergranular pores. While the actual observed chemical shift caused by the anisotropic susceptibility of graphite may vary with the specific adsorbate, it is clear that even in the case of partial graphitization, adsorbed molecules will have a negative and significantly large chemical shift with respect to their free gas counterparts. This indicates that peak $A$ is from $\mathrm{H}_{2}$ in large intergranular pores and peak $\mathrm{B}$ arises from those in much smaller pores whose population is dominated by adsorbed molecules, which is consistent with our interpreta-

(16) Mehring, M. Principles of High-Resolution NMR in Solids; SpringerVerlag: Berlin/New York, 1983; pp 25-30.

(17) Ganguli, N.; Krishnan, K. S. Proc. R. Soc. London, Ser. A, Math Phys. Sci. 1941, 177, 168-182.

(18) Tabony, J. Prog. Nucl. Magn. Reson. Spectrosc. 1980, 14, 1-26. tions in prior work ${ }^{19}$ of a similar nature. Previous studies ${ }^{20,21}$ of activated PEEK have shown the resulting materials to be predominantly microporous, so for the purposes of this work the term "micropore" will be used to describe the population of peak B, an assumption which will later be compared to the technical definition of micropores per IUPAC standards $(d<2$ $\mathrm{nm}){ }^{22}$ Since the expressions describing the behavior of the chemical shift of peak B are in addition to any bulk susceptibility effects, it is appropriate that the shift of peak A at high pressure is used as the reference shift for each data set.

3.3. Critical Pore Size. A common pore geometry in graphitic materials is the slit-shaped pore, ${ }^{23}$ and that is the structure expected to form when carbonized PEEK is activated. Using this model, eq 2 can be modified to take into account a second wall in a slit pore of width $d$ :

$$
\delta=-\lambda\left(\frac{1}{z^{3}}+\frac{1}{(d-z)^{3}}\right)
$$

where we have defined $\lambda \equiv\left|(2 / 3)(1 / 4 \pi)\left(\chi_{\|}-\chi_{\perp}\right)\right|$ for convenience, and $z$ is measured from the center of either of the walls. Figure 5 plots this function for four different values of $d$ ranging from 0.8 to $5 \mathrm{~nm}$. The black molecules represent the minimum shift produced by each slit pore, while the white molecules are "adsorbed" on the wall. In the narrower slit pores of 0.8 and $1.4 \mathrm{~nm}$ (Figure 5a,b), the walls are close enough to produce an additive effect, and thus their minimum shifts are considerably large. On the other hand, slit pores of 3 and $5 \mathrm{~nm}$ (Figure 5c,d) produce chemical shifts which are only significant near the walls and thus can be easily divided into two regions: molecules which are either positioned on the wall (i.e., adsorbed) or not. Despite appearing to be a continuous function, the chemical shift can also be treated in a similar fashion for the narrower pores. In Figure $5 \mathrm{e}$, the $1.2 \mathrm{~nm}$ slit pore is divided into discrete regions based on the monolayer thickness of $\mathrm{H}_{2}$. As in the case of wide pores, treating the shifts of the molecules as either adsorbed on one of the walls $\left(\delta_{\mathrm{ad}}\right)$ or in the free space excluded by the adsorbed monolayers $\left(\delta_{\mathrm{fg}}\right)$ is a good approximation. The notation "fg" used here is referring to "free gas", since the behavior of the $\mathrm{H}_{2}$ in the middle region of the pore is expected to be similar to such, in agreement with grand canonical ensemble Monte Carlo simulations of the particle density in a slit pore performed by Rzepka et al. ${ }^{24}$ Since the NMR time scale is too long for these subpopulations to be resolved in the spectrum, the fast exchange between the adsorbed and free species will give rise to an observed shift $\delta_{\mathrm{ob}}$ of peak B that is the weighted average of the chemical shifts of each region:

$$
\delta_{\mathrm{ob}}(P)=\frac{\delta_{\mathrm{ad}} n_{\mathrm{ad}}(P)+\delta_{\mathrm{fg}} n_{\mathrm{fg}}(P)}{n_{\mathrm{ad}}(P)+n_{\mathrm{fg}}(P)}
$$

(19) Kleinhammes, A.; Anderson, R. J.; Chen, Q.; Jeong, Y.; Chung, T. C. M.; Wu, Y. J. Phys. Chem. A 2010, submitted.

(20) Li, S. Nanostructural activated carbons for hydrogen storage. Ph.D. Thesis, State University of New York, College of Environmental Science and Forestry, Syracuse, NY, 2007.

(21) Cansado, I. P. P.; Gonçalves, F. A. M. M.; Nabais, J. M. V.; Ribeiro Carrott, M. M. L.; Carrott, P. J. M. Fuel Process. Technol. 2009, 90, 232-236.

(22) McCusker, L. B.; Liebau, F.; Engelhardt, G. Pure Appl. Chem. 2001, 73, 381-394.

(23) Texier-Mandoki, N.; Dentzer, J.; Piquero, T.; Saadallah, S.; David, P.; Vix-Guterl, C. Carbon 2004, 42, 2744-2747.

(24) Rzepka, M.; Lamp, P.; de la Casa-Lillo, M. A. J. Phys. Chem. B 1998, 102, 10894-10898. 


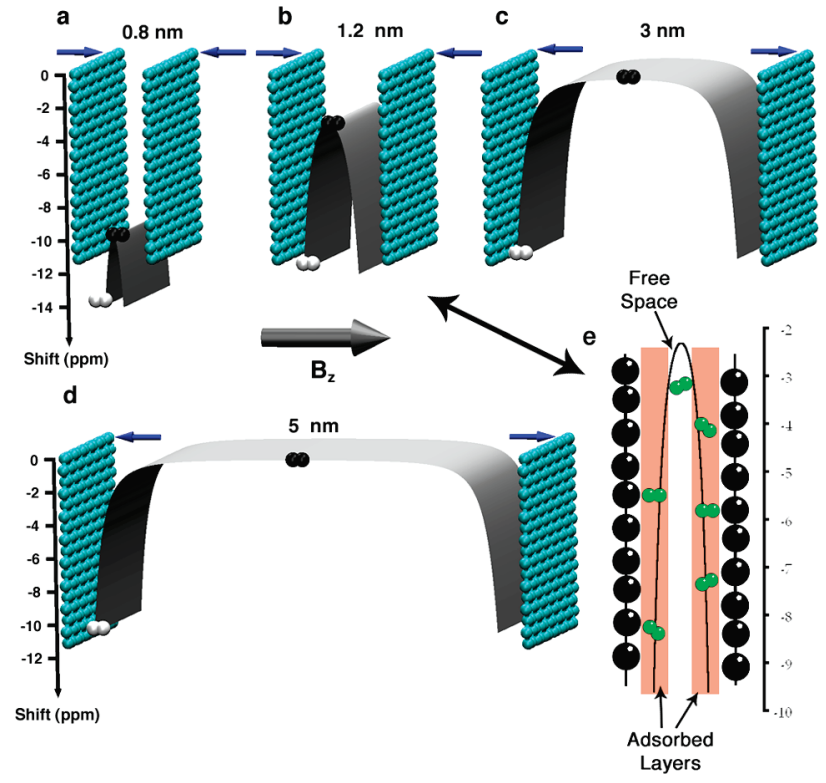

Figure 5. Surface plots of the expected chemical shift due to the ring current effect of the graphitic surfaces, as a function of distance from the left wall, for slit pores with widths of (a) 0.8 , (b) 1.2 , (c) 3 , and (d) $5 \mathrm{~nm}$. The spheres represent hydrogen molecules located on the pore wall (white) and in the middle of the slit pore (black). The minimum shift (black molecule) is appreciable for $d=0.8$ and $1.2 \mathrm{~nm}$ and has dropped to less than $-1 \mathrm{ppm}$ for $d=3 \mathrm{~nm}$; by $5 \mathrm{~nm}$ it is roughly equal to that of a free hydrogen molecule. In the $0.8 \mathrm{~nm}$ slit pore there is little distinction between the two cases. In (e) the $1.2 \mathrm{~nm}$ pore is divided into discrete regions determined by the thickness of an adsorbed monolayer of $\mathrm{H}_{2}$. Despite being a continuous function, the chemical shift in the pores can be well approximated by a free region and an adsorbed region. For all plots, $\lambda=$ $0.25 \mathrm{ppm} \mathrm{nm}{ }^{3}$, or $\left(\chi_{\|}-\chi_{\perp}\right) \approx-9.5 \times 10^{-10} \mathrm{~m}^{3} \mathrm{~mol}^{-1}$.

If the populations of these two regions evolve differently with pressure, then an effect similar to that seen in Figures 2 and 3 will appear. Following the ideal gas law, $n_{\mathrm{fg}}$ should be linear with pressure and proportional to the "free volume" (the micropore volume less the adsorbed monolayers), while the Langmuir model of adsorption describes $n_{\mathrm{ad}}$ and is given by ${ }^{25}$

$$
n_{\mathrm{ad}}(P, T)=n_{\infty} \frac{b P}{1+b P} ; \quad b=\frac{\sigma}{v_{0} \sqrt{2 \pi m_{\mathrm{H}_{2}} k_{\mathrm{B}} T}} \exp \left(\frac{E_{\mathrm{b}}}{k_{\mathrm{B}} T}\right)
$$

Here, $n_{\infty}$ is the saturation coverage, $\sigma$ is the area occupied by a $\mathrm{H}_{2}$ molecule on the surface and is about $0.16 \mathrm{~nm}^{2}$ based on the commensurate filling measurement of a graphite plane, ${ }^{26} v_{0}$ is the attempt frequency of about $10^{13} \mathrm{~Hz}, m_{\mathrm{H}_{2}}$ is the mass of $\mathrm{H}_{2}$, and $E_{\mathrm{b}}$ is the binding energy. The adsorbed shift $\delta_{\mathrm{ad}}$ is simply eq 3 with the appropriate value for $z$ of an adsorbed $\mathrm{H}_{2}$ molecule. This distance can be estimated by using the Lorentz-Berthelot mixing rules to calculate the fluid-solid Lennard-Jones (LJ) interaction parameter, $\sigma_{\mathrm{fs}}$. For hydrogen, the fluid-fluid LJ parameter is taken to be $\sigma_{\mathrm{ff}}=0.296 \mathrm{~nm},{ }^{27}$ while the value for the solid-solid LJ parameter of carbon is taken from Steele $\mathrm{e}^{28}$ to be $\sigma_{\mathrm{ss}}=0.34 \mathrm{~nm}$, producing a value of $0.318 \mathrm{~nm}$ for $\sigma_{\mathrm{fs}}$. For current purposes, it will suffice to say $z=0.32 \mathrm{~nm}$, so

(25) Boer, J. H. The Dynamical Character of Adsorption; Clarendon Press: Oxford, 1968; p 55.

(26) Nielsen, M. M.; McTague, J. P. Phys. Rev. B, Condensed Matter 1979, 19, 3096-3106.

(27) Buch, V. J. Chem. Phys. 1994, 100, 7610-7629.

(28) Steele, W. A. The Interaction of Gases with Solid Surfaces; Pergamon Press: Oxford/New York, 1974; p 56.

$$
\delta_{\mathrm{ad}}=-\lambda\left(\frac{1}{(0.32 \mathrm{~nm})^{3}}+\frac{1}{(d-0.32 \mathrm{~nm})^{3}}\right)
$$

The molecular density is very close to homogeneous in the free gas region, ${ }^{24}$ so it is assumed that an $\mathrm{H}_{2}$ molecule within this region has equal probability to be found at any point between the two adsorbed layers. To account for possible variations of the chemical shift across this region, the averaged value of eq 3 is calculated:

$$
\delta_{\mathrm{fg}}=-\lambda \frac{\int_{w}^{d-w}\left(\frac{1}{z^{3}}+\frac{1}{(d-z)^{3}}\right) \mathrm{d} z}{\int_{w}^{d-w} \mathrm{~d} z}=-\lambda \frac{d}{w^{2}(d-w)^{2}}
$$

where $w$ is the effective thickness of the pore wall excluded by the adsorbed monolayers, $\sim 0.6 \mathrm{~nm}$. For pores of width much larger than the monolayer thickness $(d \gg w), \delta_{\text {fg }}$, the chemical shift for gas within pores, approaches 0 as $1 / d$. Therefore, the observed chemical shift, $\delta_{\mathrm{ob}}(d, P)$, will change with pressure from $\delta_{\mathrm{ad}}(P=0) \sim-10 \mathrm{ppm}$ and approach $\delta_{\mathrm{fg}}(d, P=10 \mathrm{MPa})$ according to eq 4 , because at $10 \mathrm{MPa} n_{\mathrm{fg}} \gg n_{\mathrm{ad}}$ in large pores.

While it is useful to have explicit expressions for the parameters of eq 4, fitting the data in hopes of extracting a value for $d$ is complicated by the fact that there is an inhomogeneous distribution of pore sizes, and we leave this for an in-depth treatment at a later time. Comparative analysis of Steam-9-70 and CO2-9-59 shows that they have strikingly similar evolutions, and so it is suggested not only that their average pore widths are equal within experimental error, but also that they have very similar pore size distributions (this has been independently shown to be true). ${ }^{11}$ Steam-9-20 and CO2-9-26 show potential to have identical trends, but the CO2-9-26 data are too sparse to verify this.

Despite currently being limited to qualitative analysis, eq 4 can still be utilized to interpret the implications of an unchanging chemical shift. If there is no free volume within the pores, then the free gas population $n_{\mathrm{fg}}$ will equal zero for all pressures, and eq 4 will reduce to $\delta_{\mathrm{ob}}(P)=\delta_{\mathrm{ad}}$, which is assumed to be constant. It is very practical then to calculate the critical average pore size at which $\Delta \delta_{\mathrm{ob}}(P) \neq 0$ (i.e., a change in the chemical shift would be observed); those materials which exhibit only one chemical shift for peak B across the pressure range can be taken to have an average micropore size smaller than this $d_{\mathrm{c}}$. A region distinct from the adsorbed $\mathrm{H}_{2}$ could develop as soon as the average pore width increases beyond that of two closepacked monolayers of $\mathrm{H}_{2}$ and should be fairly well-defined once the pore width is equivalent to three monolayers. Following the adsorption studies of Bhatia and colleagues ${ }^{6,29,30}$ using simulations based on LJ interactions, we define a critical pore diameter range:

$$
2 l_{\mathrm{H}_{2}} \leq d_{\mathrm{c}} \leq 3 l_{\mathrm{H}_{2}} \Rightarrow 2.9 \sigma_{\mathrm{fs}} \leq d_{\mathrm{c}} \leq 3.9 \sigma_{\mathrm{fs}}
$$

where $l_{\mathrm{H}_{2}}$ is the monolayer thickness of $\mathrm{H}_{2}$ and $\sigma_{\mathrm{fs}}$ is the fluid-solid LJ interaction parameter calculated earlier. Note that the latter half of eq 8 is generalized and can be used to calculate $d_{\mathrm{c}}$ for any adsorbent-adsorbate system with monolayer adsorp-

(29) Nguyen, T. X.; Bhatia, S. K.; Nicholson, D. J. Chem. Phys. 2002, 117, 10827-10836.

(30) Bhatia, S. K.; Tran, K.; Nguyen, T. X.; Nicholson, D. Langmuir 2004, $20,9612-9620$. 


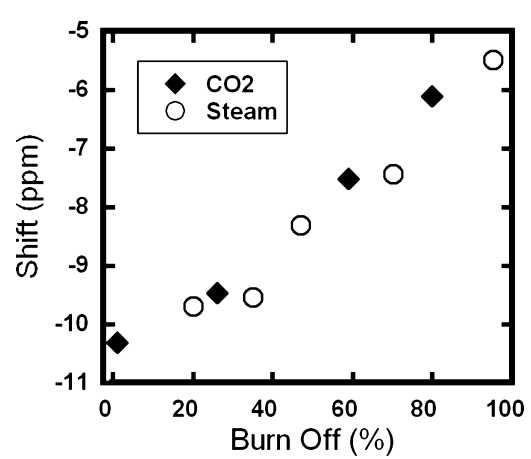

Figure 6. Chemical shift of the micropore peak at $0.1 \mathrm{MPa}$ and $100 \mathrm{~K}$ versus burnoff. The pore diameter is approximately inversely proportional to the absolute value of the shift.

tion studied by NMR. Inserting $\sigma_{\mathrm{fs}}=0.32 \mathrm{~nm}$ from above into eq 8 , we find (roughly)

$$
0.9 \leq d_{\mathrm{c}} \leq 1.2 \mathrm{~nm}
$$

Returning to Figure 3, it is noted that CO2-9-1, Steam-9-20, and CO2-9-26 all appear to have $d \leq d_{\mathrm{c}}$, while the slight downfield trend of Steam-9-35 suggests that it is just beyond the critical boundary. In general, it is probably more accurate to quote the higher value of $1.2 \mathrm{~nm}$ as the critical diameter.

3.4. Relative Pore Diameter. It is tempting to directly compare the magnitude of the shifts at a given pressure to infer the relative pore diameters of the nine samples, but in general this proves to be unreliable since it cannot be automatically assumed that the value of $\lambda$ is the same for any two given materials. It is fortunate in this case that in the low-pressure limit, where $\delta_{\mathrm{ob}} \sim \delta_{\mathrm{ad}}$, the shift of peak $\mathrm{B}$ in Figure 3 consistently approaches $-10 \mathrm{ppm}$, which also indicates that the degree of graphitization is dependent only on the argon preactivation step. This is true even in the highest case of $\mathrm{BO}$, Steam-9-95. With the value of $\lambda$ experimentally verified to be unchanged for all the data sets, the respective shift of each sample at a well-chosen pressure can be accurately used as a relative measure of pore size. A pressure of $0.1 \mathrm{MPa}$ at $100 \mathrm{~K}$ was selected because the lack of a significant contribution from peak $\mathrm{A}$ at those conditions simplified determining the value of the pore shift, as well as the fact that data at those conditions were available for all of the samples. As can be deduced from eq 7, a larger absolute shift indicates a smaller pore diameter (very roughly, $d \propto-\delta^{-1}$ ). Figure 6 plots the $0.1 \mathrm{MPa}$ shift as a function of $\mathrm{BO}$. Both methods produce a trend of increasing $d$ with increasing $\mathrm{BO}$, which should be expected.

3.5. Upper Limit of Resolvable Pores. Thus far, a lower limit and a relative comparison of the pore widths above that limit have been established. What is now needed is an approximate upper limit on which micropores peak B can represent to put Figure 6 into context, and this can be based on the resolution of the two peaks in the spectra. In order to be able to distinguish $\mathrm{H}_{2}$ in micropores from the $\mathrm{H}_{2}$ in intergranular pores, the micropores must be able to produce a shift large enough for peak B to be resolved from peak A. Our criteria for resolution will be that the absolute value of $\delta_{\mathrm{fg}}\left(d_{\max }\right)$ be on the order of (or greater than) the half-width at half-maximum (HWHM) of peak A. For the $\mathrm{CO}_{2}$ activated samples, $\overline{\mathrm{HWHM}}_{\mathrm{A}} \equiv \Delta_{\mathrm{A}} \cong 1.75$ ppm. Combining this with eqs 2 and 7 gives

$$
\left|\delta_{\mathrm{fg}}\left(d_{\max }\right)\right|=\left|\delta_{\mathrm{ad}}\right| \frac{z d_{\max }}{\left(d_{\max }-z\right)^{2}} \geq \Delta_{\mathrm{A}}
$$

where the free gas region has intentionally been extended to include the entire pore space: $w=z=0.32 \mathrm{~nm}$. The shift of an adsorbed molecule, $\delta_{\text {ad, }}$, can be determined from the low pressure limit of Figure 3, where the shifts for all nine samples have already been established as converging near $-10 \mathrm{ppm}$. Numerically evaluating eq 10 with $\left|\delta_{\text {ad }}\right|=10 \mathrm{ppm}$ and $\Delta_{\mathrm{A}}=1.75 \mathrm{ppm}$ indicates that the largest micropores that can be reliably resolved as a separate NMR peak are around $2.4 \mathrm{~nm}$. Due to the averaging process of the molecular dynamics, it is possible that slightly larger pores may be included in peak B, but $2.4 \mathrm{~nm}$ is the largest average pore size expected to produce a resolved second peak. It is clear that, even with the range of distinguishable pore sizes extending slightly beyond the micropore regime, the ability to differentiate between $\mathrm{H}_{2}$ in intergranular pores and $\mathrm{H}_{2}$ in pores with diameters on the nanometer scale makes NMR a rather useful tool when it is beneficial to be able to restrict the region of interest to the micro-/nanopore domain. The general ability to resolve the two components produced by the samples in this study is demonstrated by Figure 7, which displays the high-pressure/low-temperature spectra of all nine samples. The lone exception here is Steam-9-95, which will be discussed in more detail later. Two lines are observed for $\mathrm{H}_{2}$ in all but one sample, indicating that $\mathrm{H}_{2}$ in micropores does not exchange on the NMR time scale with $\mathrm{H}_{2}$ in intergranular pores. A 2-D exchange experiment of Steam-9-35 at RT did not show any exchange peaks and extended the time over which no exchange between the two $\mathrm{H}_{2}$ reservoirs occurs to $10 \mathrm{~ms}$.

3.6. $\mathbf{H}_{2}$ Uptake. While the intensity of the NMR signal is directly proportional to the number of protons being probed, an exact empirical relationship does not exist between the two. ${ }^{31}$ By decomposing the two peaks and properly calibrating the signal to units of $\mathrm{H}_{2}$ mass, information which is significantly more useful can be accessed. The full details of this procedure can be found in Appendix $\mathrm{C}$ in the Supporting Information. Isotherms of the weight percent (wt \%) uptake of the micropores were derived from calibrated, integrated intensities of peak B for seven of the samples and are plotted in Figure 8. Incidentally, the NMR-derived capacities are in agreement with volumetric measurements done on the same samples. ${ }^{11}$ For all samples, a maximum uptake is observed for CO2-9-59. This is clearer in Figure 9, where the uptakes at 0.2 and $10 \mathrm{MPa}$ are plotted versus BO. It can be seen that this maximal behavior is reflected at the lower pressure as well, which is a decent approximation of the traditionally reported excess uptake. For both activation methods, samples with BET surface areas of $\sim 2000 \mathrm{~m}^{2} \mathrm{~g}^{-1}$ exhibited the highest uptakes. Since the specific surface area is expected to be the main determinant of uptake, ${ }^{32,33}$ it is somewhat surprising that $\mathrm{CO} 2-9-80$, with $\sim 3000 \mathrm{~m}^{2} \mathrm{~g}^{-1}$, is inferior in this respect. It could be argued that some micropores could have zero graphitization, making them "invisible" to NMR, and therefore are not included in peak B. However, this is not quite the case and is rebutted in Appendix D in the Supporting Information. The real discrepancy between the surface area and uptake can be partially explained in terms of the structure of the material. The upper limit of the surface area of an exclusively

(31) Keeler, J. Understanding NMR Spectroscopy; Wiley: Chichester, England, 2005; p 265.

(32) Bénard, P.; Chahine, R. Scr. Mater. 2007, 56, 803-808.

(33) Panella, B.; Hirscher, M.; Roth, S. Carbon 2005, 43, 2209-2214. 


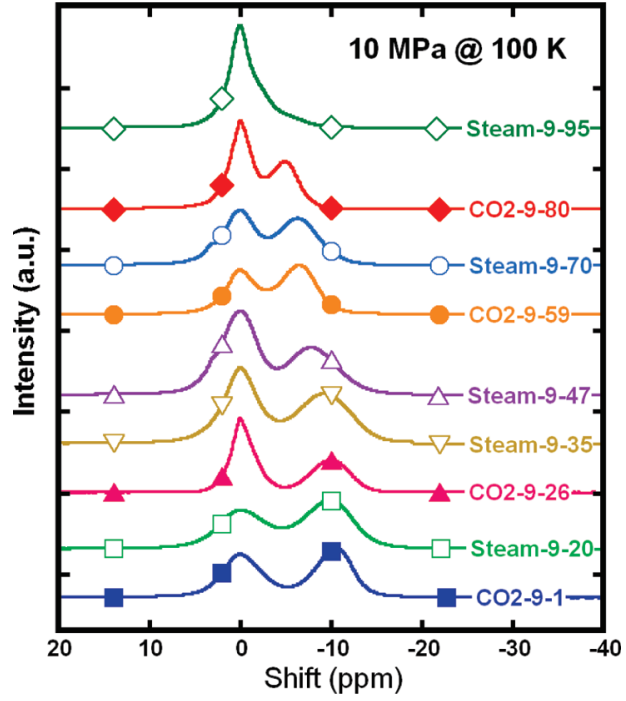

Figure 7. Non-normalized NMR spectra for all nine samples at $10 \mathrm{MPa}$ and $100 \mathrm{~K}$. The peak on the right is confirmed to be the hydrogen in the micropores (or small mesopores), while the peak on the left is hydrogen in the intergranular pores.

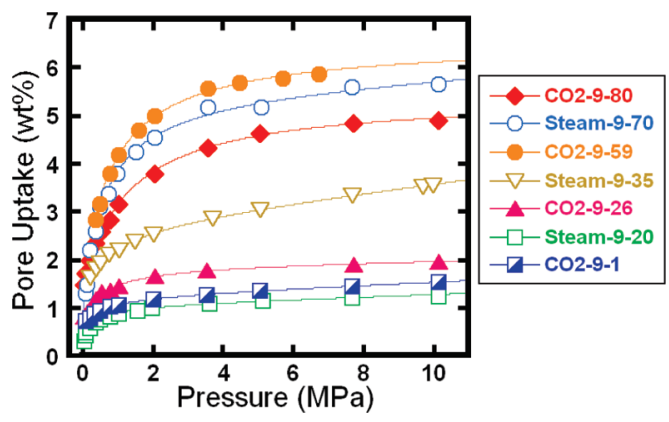

Figure 8. High-pressure $(0.1-10 \mathrm{MPa})$ isotherms at $100 \mathrm{~K}$ for seven of the nine samples with Langmuir adsorption fits.

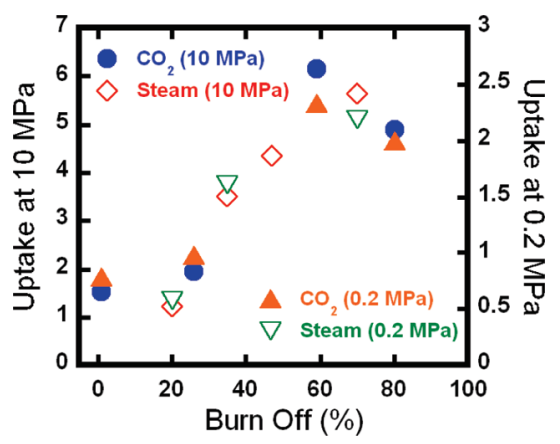

Figure 9. $\mathrm{H}_{2}$ uptake of the micropores versus burnoff at $100 \mathrm{~K}$ for 10 and 0.2 MPa. CO2-9-59 shows the highest uptake at both pressures, despite not having the largest specific surface area. This is thought to be a result of the limitations of the NMR method and an increase in the mobility between regions in the material.

slit pore geometry is the maximum theoretical surface area of both sides of a graphene sheet, $2630 \mathrm{~m}^{2} \mathrm{~g}^{-1} \cdot{ }^{34}$ Above this, the surface area must be created by deformations of the structure, which in real materials will start to appear long before 2630 $\mathrm{m}^{2} \mathrm{~g}^{-1}$ of surface area has been produced. The breakdown in the slit pore geometry at higher $\mathrm{BO}$ will produce the twin effects

(34) Peigney, A.; Laurent, C.; Flahaut, E.; Bacsa, R. R.; Rousset, A. Carbon 2001, 39, 507-514.

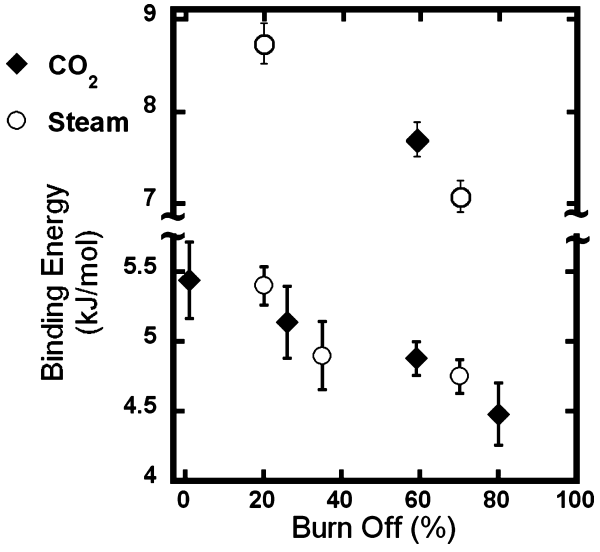

Figure 10. Binding energies extracted from high-pressure isotherms (estimated 5\% error) and full-pressure-range isotherms (estimated $2.5 \%$ error) versus burnoff for seven of the nine samples. The trend of decreasing binding energy with increasing burnoff can be correlated to the increasing widths of the pores in both cases; this is more noticeable in the higher binding energies associated with the narrower pores.

of reducing the effectiveness of the NMR method while increasing the mobility of the $\mathrm{H}_{2}$ between regions. The latter is more significant, as the small population of micropores which are in close proximity to the intergranular pores have an increasing chance of contributing to peak A than to peak B. The former is a limitation of the experiment itself, since it is possible that the higher BO samples have a sizable fraction of pores which are wider than $2.4 \mathrm{~nm}$ but still contribute to the overall uptake. Nonetheless, several theoretical studies have predicted that the pore sizes which are optimum for $\mathrm{H}_{2}$ storage are much narrower than this, ${ }^{6,24,35}$ so peak B represents $\mathrm{H}_{2}$ that can be efficiently stored from a volumetric perspective, and the maximum in its gravimetric uptake as measured by NMR at $59 \%$ BO can be viewed as a balance between these two metrics of storage capacity.

3.7. Binding Energy. The isotherms which had data for pressures below $0.1 \mathrm{MPa}$ indicated that two distinct adsorption sites were present, most likely arising from a bimodal distribution of pore sizes, with the lower binding energy site $(\sim 5 \mathrm{~kJ}$ $\mathrm{mol}^{-1}$ ) being dominant. These isotherms were fit with two Langmuir terms in the form of eq 5 and a small ideal gas term, since peak B represents all of the $\mathrm{H}_{2}$ in the micropores and not just the excess adsorption. The relevant pressure range for the lower binding energy at $100 \mathrm{~K}$ is $0.1-10 \mathrm{MPa}$, which was available for all seven samples from the previous section. In this case, a decent approximation for the fit could be made by replacing the Langmuir term with a constant offset without much loss in accuracy. Examples of low-pressure data and a comparison of the two fitting methods can be found in Appendix E in the Supporting Information. The results of the isotherm fittings are shown in Figure 10. Since the degree of graphitization of the adsorbent surface is altered very little by the activation process, the trend of decreasing binding energy is most likely due to the widening of the pores. This is consistent with the expectation that molecules in narrower pores are more likely to interact with two walls instead of just one,${ }^{36}$ leading to enhanced binding energies with decreasing pore width. Assuming that the two binding energies arise from two micropore sizes, the weaker binding energy would be assigned

(35) Wang, Q.; Johnson, J. K. J. Chem. Phys. 1999, 110, 577.

(36) Everett, D. D. H. J. Chem. Soc., Faraday Trans. 1 1976, 72, 619636. 


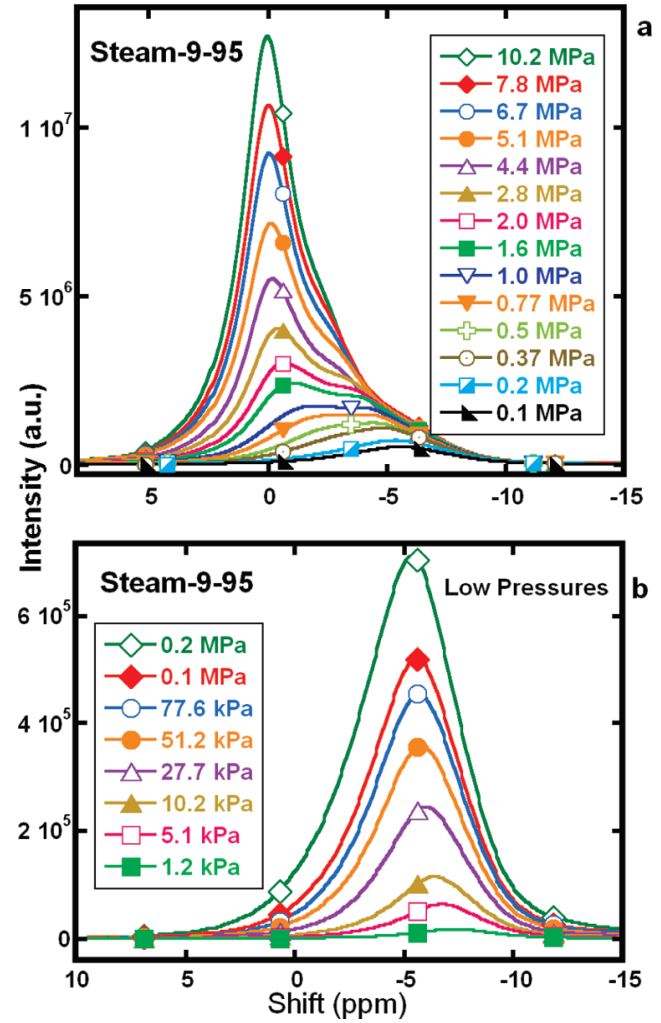

Figure 11. Pressure-dependent evolution at high pressures (a) and low pressures (b) for Steam-9-95 at $100 \mathrm{~K}$. Peak B is clearly distinct in (b) but cannot be resolved at all in (a), indicating that the average micropore size is $\sim 2.4 \mathrm{~nm}$ (or larger), the experimental upper limit of peak B. Above 1.0 MPa (a), a component due to exchange between the two regions appears between peaks A and B. This was not observed in any of the other samples.

to the larger micropores and the stronger binding energy to pores which are very likely what Dubinin ${ }^{37}$ called ultramicropores $(d$ $<0.7 \mathrm{~nm}$ ), due to the proximity to both walls which would be required to enhance the binding energy by several $\mathrm{kJ} \mathrm{mol}^{-1}$. Smaller pores are more responsive to this effect, in part explaining why the higher binding energies are more sensitive to the amount of BO than the lower binding energies.

3.8. Pore Structure at High Burnoff. Figure 11 shows the pressure-dependent spectral evolution of Steam-9-95. Peak B is distinct and clearly shifted upfield at low pressures, while at high pressures any components present in the spectra cannot be easily distinguished. Without the ability to properly decompose the spectra, it was not possible to calibrate the intensities with confidence, and therefore an isotherm for the micropore could not be produced. In the intermediate range of 1.0-4.4 $\mathrm{MPa}$, two distinct peaks are clearly present, although they would be rather difficult to accurately resolve. This indicates that the average pore size is in the neighborhood of the upper limit (2.4 $\mathrm{nm}$ ) of what our method can reliably distinguish from free gas. In the pressure regime where peak A becomes significant, there is a contribution to the spectrum between the two expected peaks which appears to be an average of their intensities and shifts, indicating that there is a considerable amount of exchange happening between the two regions on the NMR time scale. It is an exceptional case to see evidence of such exchange, as even the sample with the second highest $\mathrm{BO}$ which was studied, $\mathrm{CO} 2$ 9-80, did not show signs of exchange in its 1-D spectra (Figure

(37) Dubinin, M. M. J. Colloid Interface Sci. 1974, 46, 351-356.
7). When the ${ }^{1} \mathrm{H}$ spectra for those two samples are plotted versus a common shift reference, the dominant peak of Steam-9-95 appears between peaks A and B of CO2-9-80 (see Appendix F in the Supporting Information). This difference indicates that the structure of activated PEEK has a limiting effect on the inter-region exchange of the $\mathrm{H}_{2}$ molecules, with any perceptible inter-region exchange occurring only when its structure becomes largely disorganized in the latest stages of the BO process. However, without an accompanying increase in intra-region mobility, the inter-region exchange would be limited primarily to micropores which are directly connected to the intergranular pores. Qualitatively speaking, in Figure 11 it appears that a large number of both species participate in the exchange process, which is indicative of intercalation of the micropores throughout the sample and not just on the grain surfaces.

\section{Conclusion}

We have employed variable-pressure ${ }^{1} \mathrm{H}$ NMR using molecular hydrogen as a probing molecule to characterize the hydrogen storage capabilities and pore structures of a series of $\mathrm{CO}_{2}$ - and steam-activated PEEK samples with various amounts of burnoff. The ability to distinguish between $\mathrm{H}_{2}$ molecules occupying intergranular pores and those in micropores has enabled focused study of the $\mathrm{H}_{2}$ interaction with pores less than $\sim 2.4 \mathrm{~nm}$. The pressure-dependent behavior of the chemical shift of Peak B was shown to arise from the different pressure dependences in populations of $\mathrm{H}_{2}$ adsorbed and gaseous $\mathrm{H}_{2}$ in the micropores, which each experience a difference chemical shift due to the spatial dependence of the ring-current effect. This allowed for critical information about the average micropore diameter to be inferred, most notably whether or not it is less than an adsorbate-dependent critical diameter. No pressure-dependent shift was observed in samples with less than $35 \%$ burnoff, indicating that the average widths of their micropores were less than $1.2 \mathrm{~nm}$, and that the transition to pores wider than this occurs between 35 and $47 \%$ burnoff during activation. Values for the more common parameters of hydrogen uptake and binding energies have been extracted from $100 \mathrm{~K}$ isotherms. The degree of graphitization of the adsorbing surface appeared to be unaffected by the activation process, and thus variations in binding energy were attributed solely to micropore width. The adsorption appeared to be bimodal. Although it was not possible to accurately determine the value of the higher binding energy for samples without low pressure data, those which could be analyzed ranged from 7 to $9 \mathrm{~kJ} \mathrm{~mol}^{-1}$. The two binding energies are thought to be due to two different micropore size distributions, with the higher binding energy corresponding to ultramicropores. At $100 \mathrm{~K}$, maximum uptakes of $2.3 \mathrm{wt} \%$ at $0.2 \mathrm{MPa}$ and $6.2 \mathrm{wt} \%$ at $10 \mathrm{MPa}$ were observed for the $\mathrm{CO}_{2}$-activated sample with $59 \%$ burnoff. Increased inter-region exchange due to structure breakdown and the upper limit of resolvable pore sizes are believed to account for the apparent drop in measured uptake despite increased surface area in samples with higher burnoff. This breakdown was evidenced by the exchange between the peaks in the $10 \mathrm{MPa} / 100 \mathrm{~K} \mathrm{NMR}$ spectrum for the steam-activated sample with $95 \%$ burnoff. From a methodological point of view, we have begun to establish the NMR-H $\mathrm{H}_{2}$ approach as a valid technique for characterizing microporous graphitic materials, possibly including graphene surfaces. By complementing these methods with well-established $T_{1}$ and $T_{2}$ experiments, such as those performed 
by Riehl and Fisher, ${ }^{38}$ it may be possible to extend such studies to investigate materials such as metal-organic frameworks which are less apt to produce such upfield NMR peak shifts, if at all.

Acknowledgment. We are grateful for the support by DOE through grants DE-FC36-05GO15081 and DE-FC36-05GO15077, NSF through grant DMR-0906547, and the Carolina Energy Fellowship (R.J.A.) through funds made available by the University of North Carolina's Institute for the Environment and Institute for Advanced Materials, Nanoscience and Technology. This work was conducted under the association with the Hydrogen Sorption Center

(38) Riehl, J. W.; Fisher, C. J. J. Chem. Phys. 1973, 59, 4336-4342. of Excellence, and we thank Kevin O'Neill and Lin Simpson (NREL) for conducting the BET measurements and analysis.

Supporting Information Available: Discussions of the approximation of the ring current effect near pore walls, chemical shift averaging due to molecular dynamics, the possibility of "invisible" micropores, and isotherm fitting for two binding energies without low-pressure data; peak decomposition and intensity calibration procedure; and spectra of CO2-9-80 and Steam-9-95 plotted relative to the same reference shift. This material is available free of charge via the Internet at http:// pubs.acs.org.

JA9109924 